\title{
Genome-wide haplotype association study identifies the FRMD4A gene as a risk locus for Alzheimer's disease
}

\author{
J-C Lambert ${ }^{1,2,3,47}$, B Grenier-Boley ${ }^{1,2,3,47}$, D Harold ${ }^{4}$, D Zelenika ${ }^{5}$, V Chouraki ${ }^{1,2,3}$, Y Kamatani, ${ }^{5,6}$,
} K Sleegers ${ }^{7,8}$, MA Ikram ${ }^{9}$, M Hiltunen ${ }^{10}$, C Reitz ${ }^{11}$, I Mateo ${ }^{12}$, T Feulner $^{13}$, M Bullido ${ }^{14}$, D Galimberti ${ }^{15}$, L Concari ${ }^{16}$, V Alvarez ${ }^{17}, \mathrm{R} \mathrm{Sims}{ }^{4}, \mathrm{~A}$ Gerrish ${ }^{4}, \mathrm{~J} \mathrm{Chapman}^{4}, \mathrm{C}$ Deniz-Naranjo ${ }^{18}$, V Solfrizzi ${ }^{19}$, S Sorbi ${ }^{20}$, B Arosio ${ }^{21}$, G Spalletta ${ }^{22}$, G Siciliano ${ }^{23}$, J Epelbaum²4, D Hannequin ${ }^{25}$, J-F Dartigues ${ }^{26}$, C Tzourio ${ }^{27,28}$, C Berr ${ }^{29}$, EMC Schrijvers ${ }^{30}$, R Rogers ${ }^{11}$, G Tosto $^{11}$, F Pasquier ${ }^{3,31}$, K Bettens ${ }^{7,8}$, C Van Cauwenberghe ${ }^{7,8}$, L Fratiglioni ${ }^{32,33}$, C Graff ${ }^{33,34}$, M Delepine ${ }^{5}$, R Ferri ${ }^{35}$, CA Reynolds ${ }^{36}$, L Lannfelt ${ }^{37}$, M Ingelsson ${ }^{37}$, JA Prince ${ }^{38}, \mathrm{C}$ Chillotti ${ }^{39}$, A Pilotto $^{40}$, D Seripa ${ }^{40}$, A Boland $^{5}, M_{\text {Mancuso }}{ }^{23}, P$ Bossù $^{22}$, G Annoni ${ }^{21}$, B Nacmias ${ }^{20}, \mathrm{P}$ Bosco ${ }^{35}$, F Panza ${ }^{19}$, F Sanchez-Garcia ${ }^{18}$, M Del Zompo ${ }^{41}$, E Coto ${ }^{17}$, M Owen ${ }^{4}$, M O'Donovan ${ }^{4}, \mathrm{~F}$ Valdivieso ${ }^{14}$, P Caffara ${ }^{16}$, E Scarpini ${ }^{15}$, O Combarros ${ }^{12}$, L Buée ${ }^{2,42}$, D Campion ${ }^{25}$, H Soininen ${ }^{10}$, M Breteler ${ }^{43,44}$, M Riemenschneider ${ }^{13}, \mathrm{C}$ Van Broeckhoven ${ }^{7,8}$, A Alpérovitch $^{27,28}$, M Lathrop $^{5,6}$, D-A Trégouët ${ }^{45,46}$, J Williams ${ }^{4}$ and $P$ Amouyel ${ }^{1,2,3,31}$, EADI consortium ${ }^{48}$, GERAD consortium ${ }^{48}$

${ }^{1}$ INSERM, U744, Lille, France; ${ }^{2}$ Institut Pasteur de Lille, Lille, France; ${ }^{3}$ Université Lille-Nord de France, Lille, France; ${ }^{4}$ Medical Research Council (MRC) Centre for Neuropsychiatric Genetics and Genomics, Neurosciences and Mental Health Research Institute, Department of Psychological Medicine and Neurology, School of Medicine, Cardiff University, Cardiff, UK; ${ }^{5}$ Centre National de Genotypage, Institut Genomique, Commissariat à l'énergie Atomique, Evry, France; ${ }^{6}$ Fondation Jean DaussetCEPH, Paris, France; ${ }^{7}$ Neurodegenerative Brain Diseases group, Department of Molecular Genetics, VIB, Antwerpen, Belgium; ${ }^{8}$ Laboratory of Neurogenetics, Institute Born-Bunge, University of Antwerp, Antwerpen, Belgium; ${ }^{9}$ Departement of Epidemiology and Radiology, Erasmus MC, Rotterdam; ${ }^{10}$ Department of neurology, University of Eastern Finland and Kuopio University Hospital, Kuopio, Finland; ${ }^{11}$ G.H. Sergievsky Center, Columbia University, New York, NY, USA; ${ }^{12}$ Service of Neurology, University Hospital Marques de Valdecilla, University of Cantabria, Santander, Spain; ${ }^{13}$ Department of Psychiatry and Psychotherapy, Universitätsklinikum des Saarlandes, Universität des Saarlandes Saarbruecken, Germany; ${ }^{14}$ Centro de Biologia Molecular Severo Ochoa (UAM-CSIC) and CIBERNED, Universidad Autonoma, Madrid, Spain; ${ }^{15}$ Department of Neurological Sciences, Dino Ferrari Center, University of Milan, Fondazione Cà Granda, IRCCS Ospedale Maggiore Policlinico, Milan, Italy; ${ }^{16}$ Department of Neuroscience, University of Parma, Parma, Italy; ${ }^{17}$ Genética Molecular-Laboratorio de Medicina, Hospital Universitario Central Asturias, Oviedo, Spain; ${ }^{18}$ Servicio de Inmunología. Hospital Unviersitario de Gran Canaria Dr Negrín. Bco, Las Palmas de Gran Canaria, Spain; ${ }^{19}$ Department of Geriatrics, Center for aging brain, Memory unit, University of Bari, Bari, Italy; ${ }^{20}$ Department of neurological and psychiatric Sciences, University of Florence, Florence, Italy;

${ }^{21}$ Department of Internal Medicine, Geriatric Unit, Fondazione IRCCS Cà Granda Ospedale Maggiore Policlinico, Università degli Studi di Milano, Milan, Italy; ${ }^{22}$ Clinical and Behavioral Neurology, IRCCS Fondazione Santa Lucia, Roma, Italy; ${ }^{23}$ Neurological clinic, University of Pisa, Pisa, Italy; ${ }^{24}$ INSERM UMR 894, Paris Descartes University, Paris, France; ${ }^{25}$ Inserm U614, Faculté de Médecine-Pharmacie de Rouen, Rouen, France; ${ }^{26}$ Inserm U897, Victor Segalen University, Bordeaux, France; ${ }^{27}$ Inserm U708, Paris, France; ${ }^{28}$ UPMC University Paris 06, Paris, France; ${ }^{29}$ Inserm U1061, Hôpital La Colombière, Montpellier, France; ${ }^{30}$ Department of Epidemiology and Neurology, Erasmus MC, Rotterdam; ${ }^{31} \mathrm{CHRU}$ de Lille, Lille, France; ${ }^{32}$ Aging Reasearch Center, Department NVS, Karolinska Institutet and Stockholm University, Stockholm, Sweden; ${ }^{33}$ Department of Geriatric Medicine, Karolinska University Hospital, Stockholm, Sweden; ${ }^{34} \mathrm{KI}$-Alzheimer's Disease Reseach Center, Department NVS, Karolinska Institutet, KIADRC, Stockholm, Sweden; ${ }^{55}$ IRCCS Associazione Oasi Maria SS, Institute for Research on Mental Retardation and Brain Aging, Troina (EN), Italy; ${ }^{36}$ Department of Psychology, University of California at Riverside, Riverside, CA, USA; ${ }^{37}$ Department of Public health and Caring Sciences, Rudbeck Laboratory, Uppsala University, Uppsala, Sweden; ${ }^{38}$ Department of Medical Epidemiology and Biostatistics, Karolinska Institute, Stockholm, Sweden; ${ }^{39}$ Unit of Clinical Pharmacology, Teaching Hospital of Cagliari, Cagliari, Italy; ${ }^{40}$ Geriatric Unit \& Gerontology-Geriatrics Research Laboratory, Department of Medical Sciences, I.R.C.C.S. 'Casa Sollievo della Sofferenza', San Giovanni Rotondo, Italy; ${ }^{41}$ Section of Clinical Pharmacology, Department of Neurosciences 'B.B. Brodie', University of Cagliari, Cagliari, Italy; ${ }^{42}$ Inserm U837, Lille, France; ${ }^{43}$ Department of Epidemiology, Erasmus MC, Rotterdam; ${ }^{44}$ DZNE, Bonn, Germany; ${ }^{45}$ INSERM UMR_S 937, Paris, France and ${ }^{46}$ ICAN Institute of Cardiometabolism and Nutrition, Université Pierre \& Marie Curie, Paris, France

Correspondence: Dr J-C Lambert, INSERM U744, Institut Pasteur de Lille, BP 245, 1 rue du professeur Calmette, 59019 Lille cedex, France.

E-mail: jean-charles.lambert@pasteur-lille.fr

${ }^{47}$ These authors contributed equally to this work.

${ }^{48} \mathrm{~A}$ full list of members is provided in the Supplementary Note.

Received 15 November 2011; revised 3 January 2012; accepted 10 January 2012; published online 20 March 2012 
Recently, several genome-wide association studies (GWASs) have led to the discovery of nine new loci of genetic susceptibility in Alzheimer's disease (AD). However, the landscape of the AD genetic susceptibility is far away to be complete and in addition to single-SNP (single-nucleotide polymorphism) analyses as performed in conventional GWAS, complementary strategies need to be applied to overcome limitations inherent to this type of approaches. We performed a genome-wide haplotype association (GWHA) study in the EADI1 study ( $n=2025$ AD cases and 5328 controls) by applying a sliding-windows approach. After exclusion of loci already known to be involved in AD (APOE, BIN1 and CR1), 91 regions with suggestive haplotype effects were identified. In a second step, we attempted to replicate the best suggestive haplotype associations in the GERAD1 consortium (2820 AD cases and 6356 controls) and observed that 9 of them showed nominal association. In a third step, we tested relevant haplotype associations in a combined analysis of five additional casecontrol studies (5093 AD cases and 4061 controls). We consistently replicated the association of a haplotype within FRMD4A on Chr.10p13 in all the data set analyzed (OR: $1.68 ; 95 \% \mathrm{Cl}$ : (1.43-1.96); $\left.P=1.1 \times 10^{-10}\right)$. We finally searched for association between SNPs within the FRMD4A locus and AB plasma concentrations in three independent non-demented populations $(n=2579)$. We reported that polymorphisms were associated with plasma $A \beta 42 /$ A 340 ratio (best signal, $P=5.4 \times 10^{-7}$ ). In conclusion, combining both GWHA study and a conservative three-stage replication approach, we characterised FRMD4A as a new genetic risk factor of $A D$.

Molecular Psychiatry (2013) 18, 461-470; doi:10.1038/mp.2012.14; published online 20 March 2012

Keywords: Alzheimer; amyloid; FRMD4A; GWAS; plasma

\section{Introduction}

The identification of genes involved in monogenic forms of Alzheimer's disease (AD) has significantly contributed to our knowledge of the disease mechanisms. The causal links between mutations, the functions of the mutated genes (APP, PS1 and PS2) and disease development prompted a pathophysiological hypothesis, which radically changed our understanding of $\mathrm{AD}$ : the amyloid cascade hypothesis. ${ }^{1}$ The systematic association of pathogenic mutations with changes in APP metabolism and, more particularly, a relative overproduction of $A \beta 42$ peptides indicates that this metabolism is at the heart of the disease process (at least in the monogenic forms of the disease). The overproduction of these neurotoxic peptides is supposed to lead to or accentuate neuron-to-neuron propagation of the $\tau$ pathology (leading to neuronal death) by an unknown mechanism. ${ }^{2}$

By analogy, it was expected that the characterization of genetic factors involved in the common forms of $\mathrm{AD}$ (that is, lacking classical Mendelian inheritance), the most frequent form of the disease, should also help to better understand the $\mathrm{AD}$ physiopathological process. However, the characterization of these genetic factors has encountered significant difficulties. Until 2009, the APOE (apolipoprotein E) gene was the only globally valid genetic determinant of $\mathrm{AD}$ to have been unambiguously identified in 15 years of intensive research. ${ }^{3,4}$

As with other multifactorial diseases, this systematic inability to detect new genetic determinants has prompted more comprehensive investigations using genome-wide association studies (GWASs). We and others performed five large GWASs in this field and reported that the $C L U$ (clusterin), PICALM (phosphatidylinositol-binding clathrin assembly protein), CR1 (complement component (3b/4b) receptor 1), BIN1 (bridging integrator 1), ABCA7 (ATPbinding cassette, sub-family A, member 7), MS4A (membrane spanning 4A) cluster, EPHA1 (ephrin typeA receptor 1), CD33 (differentiation antigen 33) and $C D 2 A P$ (CD2-associated protein) genes were associated with the $\mathrm{AD}$ risk. ${ }^{5-9}$ Most of these susceptibility genes have been already systematically replicated in Caucasians in large case-control studies and in families. ${ }^{10}$

However, our understanding of the $\mathrm{AD}$ genetics is far away to be complete and strong efforts have still to be done. At this level, classical GWAS approaches present an important limitation with systematic application of a conventional, highly conservative Bonferroni correction leading to select only the most statistically significant associations (commonly, $P<1 \times 10^{-8}$ ). This involves the risk of rejecting biologically valid hypotheses on purely statistical grounds, that is, false negatives.

To partly handle some of this limitation, other complementary approach consists in extracting pertinent information from single-nucleotide polymorphisms (SNPs) nominally associated with the risk of developing AD in GWAS by using complex statistical and bioinformatics multiple-SNP analyses such as genome-wide haplotype association (GWHA) study. ${ }^{11}$ We adopted this GWHA strategy to the AD genetic susceptibility through a three-step approach.

\section{Materials and methods}

\section{Population description}

The main characteristics of the populations used for the GWHA study are described in the Supplementary 
Notes and Supplementary Table 1. All AD cases met the criteria for either probable AD (NINCDS-ADRDA, DSM-IV) ${ }^{12}$ or definite AD (CERAD). ${ }^{13}$ All elderly controls were screened for dementia using the MMSE or the AD Assessment Scale-cognitive subscale and were determined to be free from dementia at neuropathological examination or had a Braak score of 2.5 or below. All subjects or, in those with substantial cognitive impairment, a caregiver, legal guardian, or other proxy gave written informed consent for participation in this study. The study protocols for all populations were reviewed and approved by the appropriate Institutional review boards of each country.

The main characteristics of the populations (nondemented individuals) used for the $A \beta$ plasma study (3C study, Rotterdam and CHS) are described in the Supplementary Notes and Supplementary Table $2 .{ }^{14-16}$

\section{Genotyping}

Participants in the French GWA study (including the 3C study) were genotyped using an Illumina 610quad array (Illumina, San Diego, CA, USA). Quality control and analytical parameters have been described in detail elsewhere. ${ }^{5}$ The GERAD participants were genotyped using an Illumina 610-quad array (Illumina), a HumanHap550 array or a HumanHap300 array (Illumina). Again, QC and analytical parameters have been described in detail elsewhere. ${ }^{6}$ The Rotterdam and CHS studies were genotyped using the Affymetrix 500K array (Affymetrix, Santa Clara, CA, USA). Again, QC and analytical parameters have been described in detail elsewhere. ${ }^{7}$

In the European populations (stage 3), genotyping was performed using Sequenom assays at the exception of the German population genotyped with a $610 \mathrm{~K}$ ILLUMINA chip (Illumina). The primer and probe sequences used in the genotyping assays are available upon request. In order to avoid any genotyping bias, cases and controls were randomly mixed while genotyping and laboratory personnel were blinded to case/control status. The genotyping success rate was at least $95 \%$. Departure from Hardy-Weinberg equilibrium was observed for rs2446581 in the Swedish control and case samples $\left(P=9.1 \times 10^{-7}\right.$ for the whole population). The Swedish sample was consequently excluded from further analysis, as haplotype analyses that do not comply with Hardy-Weinberg equilibrium are likely to bias observations.

\section{Statistical analyses}

Missing age or gender data. Any individuals with missing age or gender data were excluded. This gave a maximum of $2025 \mathrm{AD}$ cases and 5328 controls in step 1, $2820 \mathrm{AD}$ cases and 6356 controls in step 2 and 5093 $\mathrm{AD}$ cases and 4061 controls in step 3.

Detection of haplotype effects using a sliding-window approach. This approach has been fully described elsewhere. ${ }^{11,17}$ Briefly, the search for haplotype effects was carried out by applying a sliding-window approach $^{18,19}$ to the French GWA data set for each chromosome. After excluding SNPs not in HardyWeinberg equilibrium or with a minor allele frequency $(\mathrm{MAF})<0.02$, the first step of the strategy was to eliminate part of the redundancy between SNPs by using haplotype-tagging SNPs (htSNPs). For this, the same binning procedure as in ${ }^{11}$ was used: within each bin of 10 adjacent SNPs, we identified a minimal set of htSNPs that were able to characterize more than $95 \%$ of the inferred haplotypes with estimated frequency greater than 0.02 . Once a bin had been characterized by a set of htSNPs, the same strategy was applied to the bin composed of the next 10 adjacent SNPs. The final set of htSNPs $(n=287956)$ was then fed into the sliding-windows approach.

Given a window of $10 \mathrm{htSNPs}$, the search for the most informative and parsimonious haplotype configuration in terms of disease prediction was performed for all possible 1 to 4 loci combinations of not necessarily adjacent SNPs. We used a strategy based on Akaike's information criterion, which has been previously described for candidate gene haplotype analysis. ${ }^{19,20}$ It relies on the stochastic expectation-maximisation module ${ }^{21}$ in THESIAS software. ${ }^{22}$ If required, missing genotypes were inferred by applying, ${ }^{23}$ multiple imputation. ${ }^{21}$ In all, 37330050 combinations were investigated in our genome scan and this investigation was conducted thanks to the use of the grid technology developed by the European Grid Infrastructure (http://www.egi.eu). ${ }^{20}$ This technology enables several thousand computations to be run in parallel on a large number of different CPUs. The sliding-window haplotype approach was developed into a GridHaplo grid package for the EGI grid (http://genecanvas.ecgene.net). ${ }^{11}$

Replication of haplotype effects. Regions with 'window $P$-values' (i) below $10^{-5}$ and (ii) 100 times smaller than the smallest single-locus $P$-value (including all SNPs and not only htSNPs) were analyzed in terms of replication in the GERAD1 data set by using THESIAS software, with systematic adjustment for age and gender.

Association of FRMD4A SNPs with A $\beta$ plasma concentrations. In each center, $A \beta$ plasma variables are normally distributed. We excluded prior analyses all samples with a $+/-$ two s.d. values in order to avoid potential associations driven by extreme observations. Finally, each quantitative variable was transformed into a $Z$-score (equal to (observed value minus the sample mean), divided by the sample s.d.). The association between the $A \beta_{1-40}, A \beta_{1-42}$ and $\mathrm{A} \beta_{1-42} / \mathrm{A} \beta_{1-40}$ z-scores on one hand and imputed FRMD4A imputed SNPs on the other (see below) were assessed using a general linear model under an additive model adjusted for age, center and gender.

We used inverse-variance weighting (also known as fixed-effects meta-analysis) to investigate the homogeneity of haplotype effects from one study 
to another and to provide meta-analysed, age- and gender-adjusted ORs for haplotype effect estimates in the seven studies. A similar strategy was used to provide meta-analysed, age- and gender-adjusted association levels between $A \beta$ plasma $z$-scores and FRMD4A SNPs.

\section{Imputation analyses}

We imputed SNPs by using MaCH (http://www.sph. umich.edu/csg/abecasis/mach/index.html) and minimac software (http://genome.sph.umich.edu/wiki/ minimac). The reference haplotype data are provided by the MaCH website, which was built for the combined Caucasian populations as part of the 1000 genomes project. In our data set, all individuals were genotyped on the same platform (the Illumina Human660W-Quad Beadchip) and we used 492941 observed SNP genotypes that passed quality filters as follows: genotyping call rate $\geqslant 98 \%$, Hardy-Weinberg Equilibrium Test $P$ value $\geqslant 1 \times 10^{-6}$ and $\mathrm{MAF} \geqslant 1 \%$. We first inferred haplotype combinations of each individual using the 'phase' option in the $\mathrm{MaCH}$ program and then imputed them with minimac. As minimac is a newly developed software tool, we compared the correlation between the imputed genetic dosage from minimac and those from the standard $\mathrm{MaCH}$ program for SNPs in chromosome 22. The results were very similar (data not shown). Doses for 7704555 million SNPs with a MAF $>0.01$ were available from the French GWA data set using the 1000 genomes data set. We selected 2538 SNPs within the Chromosome 10p13 locus of interest (chromosome10: $13655705-14402866$ ) and evaluated their associations with $\mathrm{AD}$ risk in an additive logistic regression model adjusted for age, gender and disease status. A graphic representation was then generated with Locuszoom software (http://csg.sph.umich.edu/locuszoom/).

In the 3C, Rotterdam and CHS cohorts, we used the genotype data to impute to the 2.5 million non-monomorphic, autosomal SNPs described in
HapMap II (CEU population) as described elsewhere. ${ }^{7}$ We selected 1486 SNPs (MAF $>0.01$ ) within the Chromosome 10p13 locus of interest (chr10:13 655 70514402866 ) and evaluated their associations with A $\beta$ plasma concentrations as described above. Again, a graphic representation was then generated with Locuszoom software (http://csg.sph.umich.edu/locuszoom/).

\section{Results}

We developed a three-step approach. In the first step, the French GWA study (EADI1 for European Alzheimer's initiative 1), ${ }^{5}$ including $2025 \mathrm{AD}$ cases and 5328 controls was used to select regions with potential haplotype associations with AD. Following a slidingwindows approach (see Material and methods), we applied two a priori criteria to select loci of interest as previously described: ${ }^{11}$ level of association with a $P$-value (i) below $10^{-5}$ and (ii) at least 100 times smaller than the smallest single-SNP $P$ value observed in the corresponding locus. We were able to detect loci already known to be involved in $\mathrm{AD}$ (the $A P O E$, BIN1 and CR1 locus) ${ }^{5-9}$ from previous GWASs. The obtained signal was systematically highly stronger than the one observed for each SNP taken separately (Table 1). After exclusion of these loci, we retained 91 regions of interest.

In the second step, we replicated these haplotype associations in the GWA database from another consortium involved in the study of $\mathrm{AD}$ genetic susceptibility, the GERAD1 consortium, ${ }^{6}$ including $2820 \mathrm{AD}$ cases and 6356 controls. All 91 regions were available for investigation and 9 of them showed nominal association $(P<0.05$; Table 2). We decided to further investigate two regions showing the same best haplotypes associated with $\mathrm{AD}$ risk in both EADI1 and GERAD1, with similar magnitude and direction of association (Table 2). These two loci, not previously detected in single-SNP GWAS analyses, were located on chromosomes 6p21 and 10p13.

Table 1 Best haplotype combination observed in CR1, BIN1 and APOE and comparison with the association obtained for single-SNP analysis within these loci

\begin{tabular}{|c|c|c|c|c|c|c|}
\hline \multirow[t]{2}{*}{ Locus } & \multirow[t]{2}{*}{ hTag-SNPS } & \multirow[t]{2}{*}{$\begin{array}{l}\text { Global haplotype } \\
\text { P-value }\end{array}$} & \multicolumn{2}{|c|}{$\begin{array}{l}\text { Best associated } \\
\text { hTag-SNP }\end{array}$} & \multicolumn{2}{|c|}{$\begin{array}{l}\text { Best associated SNP } \\
\text { within the locus }\end{array}$} \\
\hline & & & $\begin{array}{c}R S \\
\text { number }\end{array}$ & $\begin{array}{l}\text { Single P-value } \\
\text { for hTag-SBP }\end{array}$ & $\begin{array}{c}R S \\
\text { number }\end{array}$ & $\begin{array}{c}\mathrm{P} \text { value_single } \\
(C N G)\end{array}$ \\
\hline CR1 & $\begin{array}{l}\text { rs3818361 + rs1323720 + } \\
\text { rs7527798 + rs2761424 }\end{array}$ & $4.12 \mathrm{E}-10$ & rs3818361 & $2.6 \mathrm{E}-06$ & rs3818361 & $2.6 \mathrm{E}-06$ \\
\hline BIN1 & $\begin{array}{l}\text { rs17014873 + rs749008 + } \\
\text { rs13031703 + rs744373 }\end{array}$ & $3.13 \mathrm{E}-09$ & rs744373 & $1.04 \mathrm{E}-03$ & rs10194375 & $2.4 \mathrm{E}-04$ \\
\hline APOE & $\begin{array}{l}\text { rs } 157580+\text { rs8106922 } \\
\text { rs405509+ rs439401 }\end{array}$ & $9.51 \mathrm{E}-184$ & rs157580 & $5.4 \mathrm{E}-32$ & rs2075650 & $3.32 \mathrm{E}-126$ \\
\hline
\end{tabular}

Abbreviations: APOE, apolipoprotein E; BIN1, bridging integrator 1; CR1, complement component (3b/4b) receptor 1; hTag-SNPs, haplotype-tagging SNPs; SNP, single-nucleotide polymorphism. 


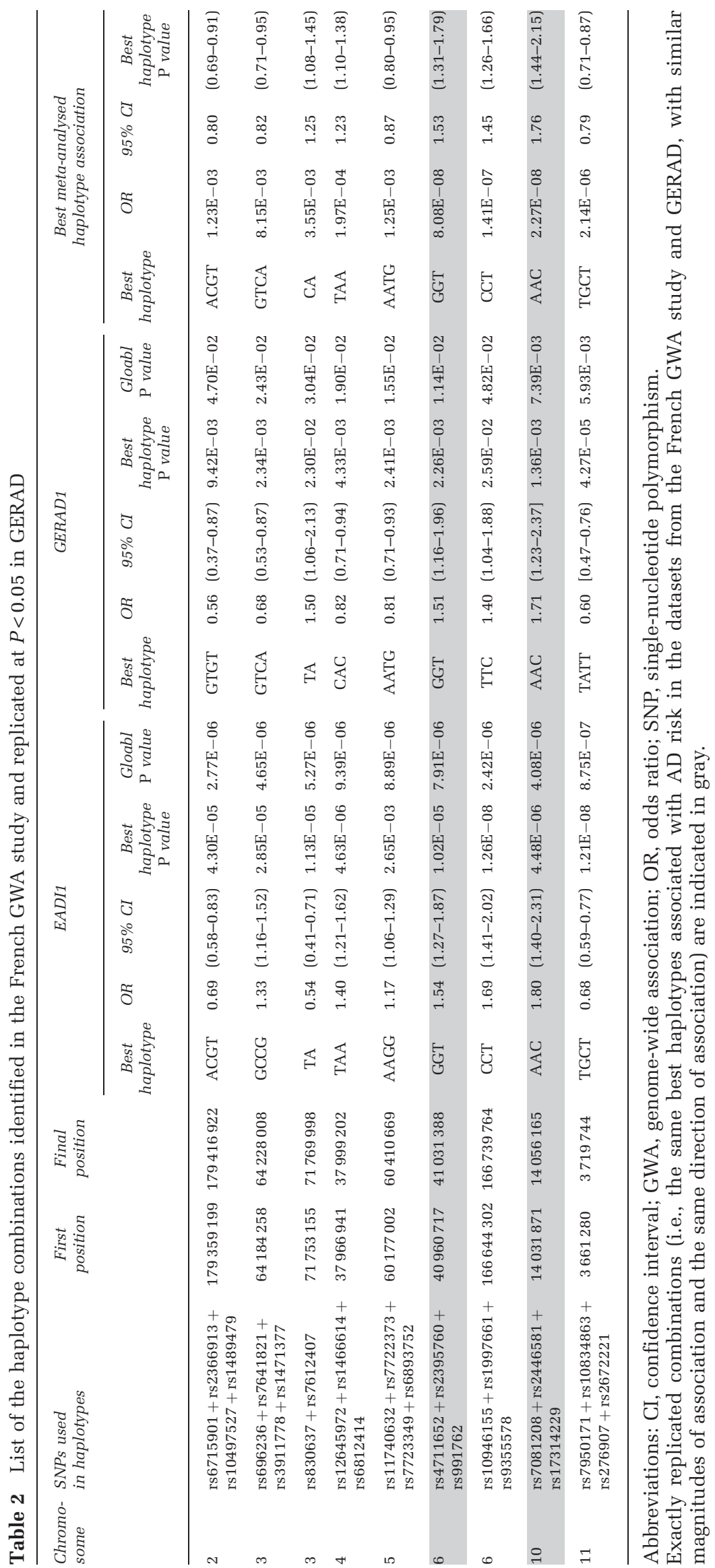


The identified AD-associated haplotypes at the 6 p21 region were tagged by rs2395760, rs991762 and rs4711652. The rs7081208, rs2446581 and rs17314229 tagged the 10p13 haplotypes. For the Chromosome 6p21 locus, the best association was attributable to the GGT haplotype in both GWASs (OR (Odds Ratio): 1.53; 95\% CI: (1.31-1.79); $P=8.1 \times 10^{-8}$ after adjustment for age and gender when both EADI1 and GERAD1 studies were combined). For the Chromosome 10p13 locus, the highest level of association was attributable to the AAC haplotype (OR: 1.76; 95\% CI: (1.44-2.15); $P=2.3 \times 10^{-8}$ after adjustment for age and gender when both EADI1 and GERAD1 studies were combined. Additional adjustment for the four main principal components did not modify the results, data not shown) (Table 2).

In the third step, the six tagging SNPs were further genotyped in five additional AD case-control studies from Flanders-Belgium (842 cases and 489 controls), Finland (560 cases and 623 controls), Germany (728 cases and 961 controls), Italy (1846 cases and 904 controls) and Spain (1117 cases and 1084 controls) (Supplementary Tables 3 and 4). The same common haplotypes were inferred from the six SNPs in the two loci, with the exception of the AGT haplotype in Chromosome 10p13 in the Italian population (frequency $<0.01$ in controls) (Supplementary Table 5 and 6).

In a combined analysis of the five replication data sets, the overall difference in haplotype distribution for the 6p21 locus was not significant between AD cases and controls $(P=0.13)$ and the GGT haplotype was not associated with AD risk (OR: 0.89; 95\% CI: (0.74-1.08); $P=0.23$ after adjustment for age and gender). We therefore considered that the haplotype association in this locus was not confirmed (see Supplementary Table 5).

Conversely, an overall significant difference in haplotype distribution between AD cases and controls was observed at the $10 \mathrm{p} 13$ locus in the replication sample $\left(P=4.3 \times 10^{-2}\right.$ after adjustment for age, gender and country) and the AAC haplotype was associated with increased $\mathrm{AD}$ risk in the five data sets meta-analysis (OR: 1.55; 95\% CI: (1.19-2.00); $P=9.2 \times 10^{-4}$ after adjustment for age and gender). When the seven data sets (EADI1, GERAD1 and the follow-up studies) were analyzed together, the AAC haplotype had a meta-analysed OR of 1.68 (95\% CI: (1.43-1.96); $\quad P=1.1 \times 10^{-10}$ adjusted for age and gender) when compared with the most frequent GGC haplotype, with no evidence of heterogeneity across the seven countries $(P=0.92)$ (Figure 1). Among the whole sample, nine cases were homozygous for the AAC haplotype versus only five controls (OR: 2.85; 95\% CI: (0.88-9.76); $P=0.09$ with Yates correction), which is consistent with a dose-dependent effect of the AAC haplotype.

Importantly, none of the individual SNPs were associated with $\mathrm{AD}$ risk in the total sample with a $P$ value lower than $10^{-5}$. The best meta-analysed SNP was rs2446581 (OR: 1.15; 95\% CI: (1.08-1.24); $P=2.1 \times 10^{-5}$ after adjustment for age and gender; Supplementary Table 7). The hypothesis that the rs2446581 could solely explain the association observed with the 10p13 haplotypes was rejected by use of the likelihood ratio test $\left(P=9.1 \times 10^{-7}\right)$.

We also tested whether this haplotype association might be explained by one or more untyped SNPs located nearby the genotyped SNPs. Genotypes for 7704555 million SNPs with a $\mathrm{MAF} \geqslant 0.01$ were imputed from the French GWA data by using the 1000 genome dataset (http://www.1000genomes. org/). None of the single SNPs imputed at this locus $(n=2538)$ showed stronger evidence of association with the $\mathrm{AD}$ risk than the haplotypes initially identified (Supplementary Figure 1). The 10p13 haplotype region appeared to be fully included

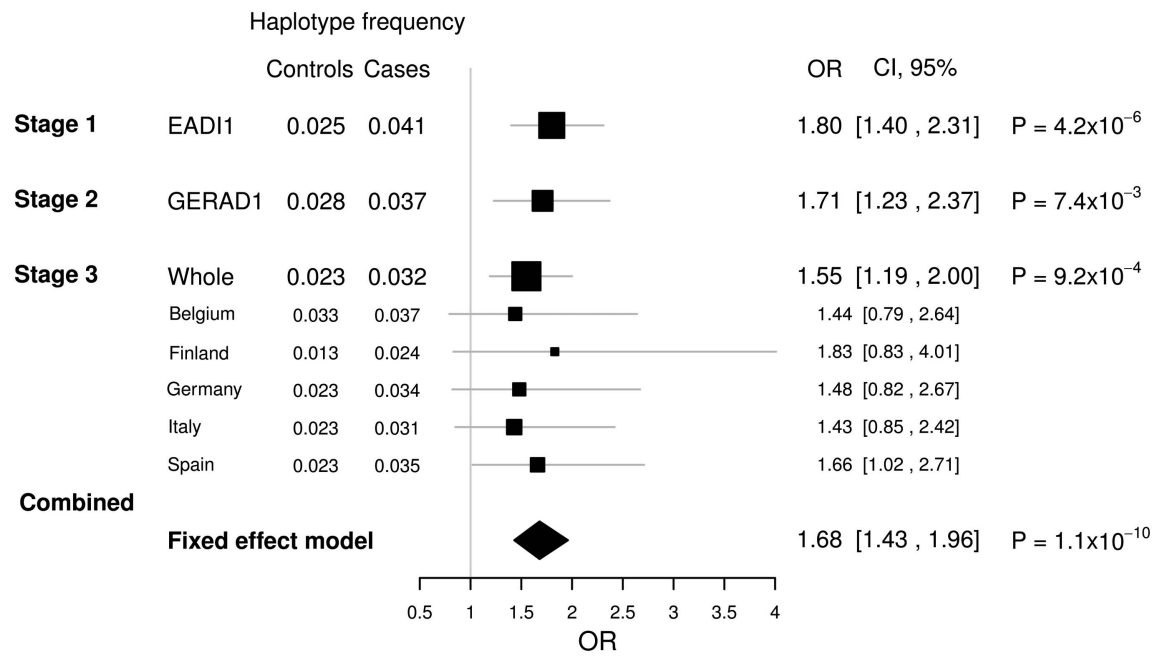

Figure 1 Haplotypic Odd ratios (ORs) for Alzheimer's disease (AD) risk with the AAC haplotype derived from rs7081208, rs2446581, rs17314229 at Chromosome 10p13 in seven independent European populations. 


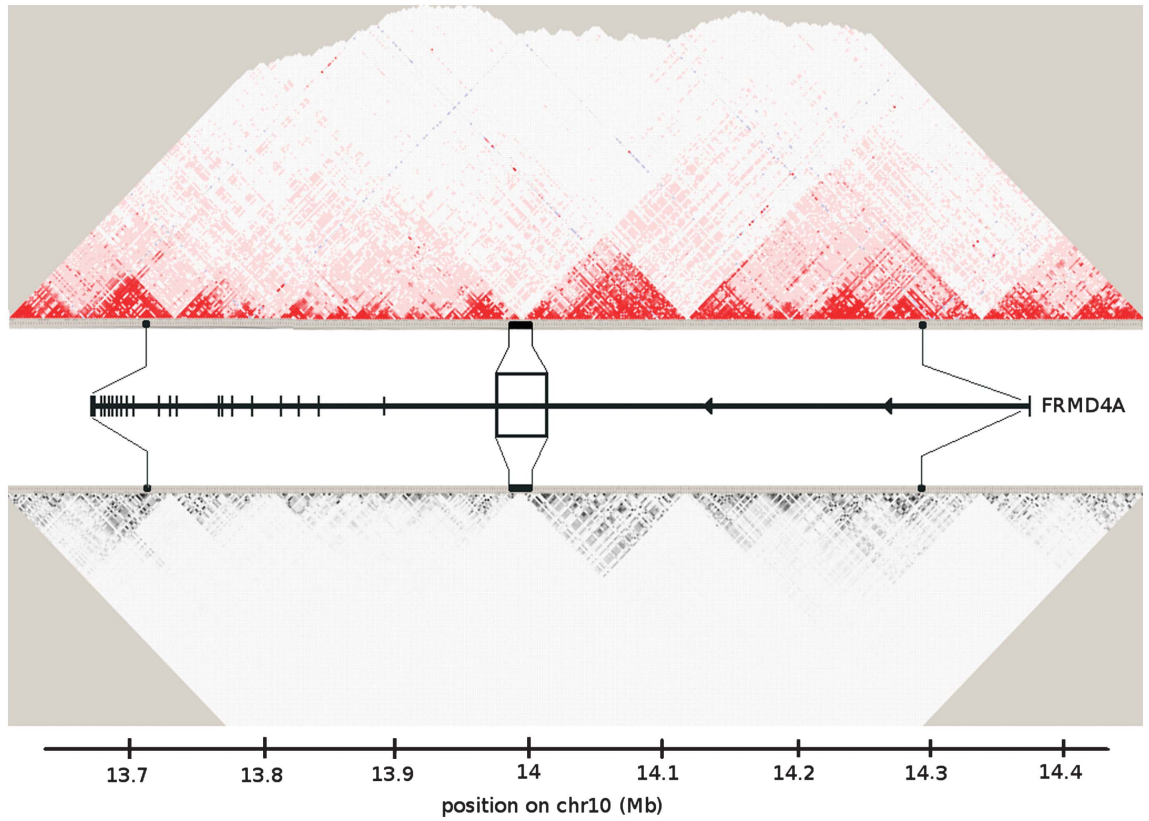

Figure 2 Linkage disequilibrium map in the FRMD4A locus and localization of the region defined by the rs7081208, rs2446581 and rs17314229 at this locus (in bold).

within the FERM domain containing 4A (FRMD4A) gene, as indicated by the linkage disequilibrium map of this locus of interest (Figure 2).

We finally explored how FRMD4A might be involved in the $\mathrm{AD}$ process. According to a recent report, the FRMD4A gene was described to interact with Arf6. ${ }^{24}$ As this latter protein was reported to control APP processing, ${ }^{25}$ we postulated that the FRMD4A locus could be associated with endophenotypes susceptible to reflect modulation of the APP metabolism. We accordingly analyzed association of the FRMD4A locus with $A \beta$ peptide plasma concentrations in three independent populations of nondemented individuals $(n=2579) \quad$ (Supplementary Table 2) for which FRMD4A imputed SNPs, $A \beta_{1-40}$ and $A \beta_{1-42}$ plasma concentrations were available. Strong associations were observed between several FRMD4A SNPs and $\mathrm{A} \beta_{1-42} / \mathrm{A} \beta_{1-40}$ (nine SNPs reaching a significant level after Bonferroni correction, $P=3.4 \times 10^{-5}$ for 1486 SNPs, Figure 3 ). The best signal was obtained for rs7921545 (meta-analysed $z$-score $\beta$ coefficient: 0.12, CI: 95\% (0.07-0.017); $P=5.4 \times 10^{-7}$ ) and was homogeneous between the three data sets $\left(P\right.$ for heterogeneity $=3.7 \times 10^{-1}$, Supplementary Figure 2). Of note, rs2446581 showed nominal association with $\mathrm{A} \beta_{1-42} / \mathrm{A} \beta_{1-40}\left(P=3.1 \times 10^{-2}\right.$, see Supplementary Figure 2). Furthermore, only nominal associations between FRMD4A SNPs, $\mathrm{A} \beta_{1-40}$ or $\mathrm{A} \beta_{1-42}$ were detected (see Supplementary Figure 3).

\section{Discussion}

From a specific haplotype-based GWAS approach, we were able to detect a new genetic susceptibility factor for $\mathrm{AD}$ that could not be identified through usual GWAS analyses. Owing to the high number of association tests performed in this GWHA study (37330050, not all of which were independent), a robust replication strategy was necessary. Our threestep approach was thus particularly conservative and we cannot rule out the possibility that we failed to identify other haplotype-based loci associated with AD risk. Nonetheless, our GWHA immediately identified two potential AD susceptibility loci among which the 10p13 locus showed an AAC haplotype that was strongly and consistently associated with $\mathrm{AD}$ risk in seven independent European populations. Interestingly, this locus is included in the large AD linkage region regularly identified on chromosome $10 .^{26}$

Additional work will be necessary to determine whether the $\mathrm{AD}$ risk-haplotype association indicates an interaction between SNPs or whether they tag nongenotyped, functional variants. However, our GWHA study could have handled several of the inherent limitations of GWAS. As the AAC haplotype is rare (with a mean frequency of $2 \%$ in our Caucasian populations), our work suggests the possibility that rare variants may be responsible for the signal detected within the FRMD4A gene. This might explain why the locus was not detected (i) in our previous GWA studies based on single-SNPs analyses $^{5-9}$ and (ii) through imputation, as SNPs with low frequency and/or SNPs within specific regions with low LD are poorly imputed even when using the 1000 genome data set (Figure 2). Furthermore, we cannot rule out the possibility that the AAC haplotype tags insertion-deletion variants or copy-number 


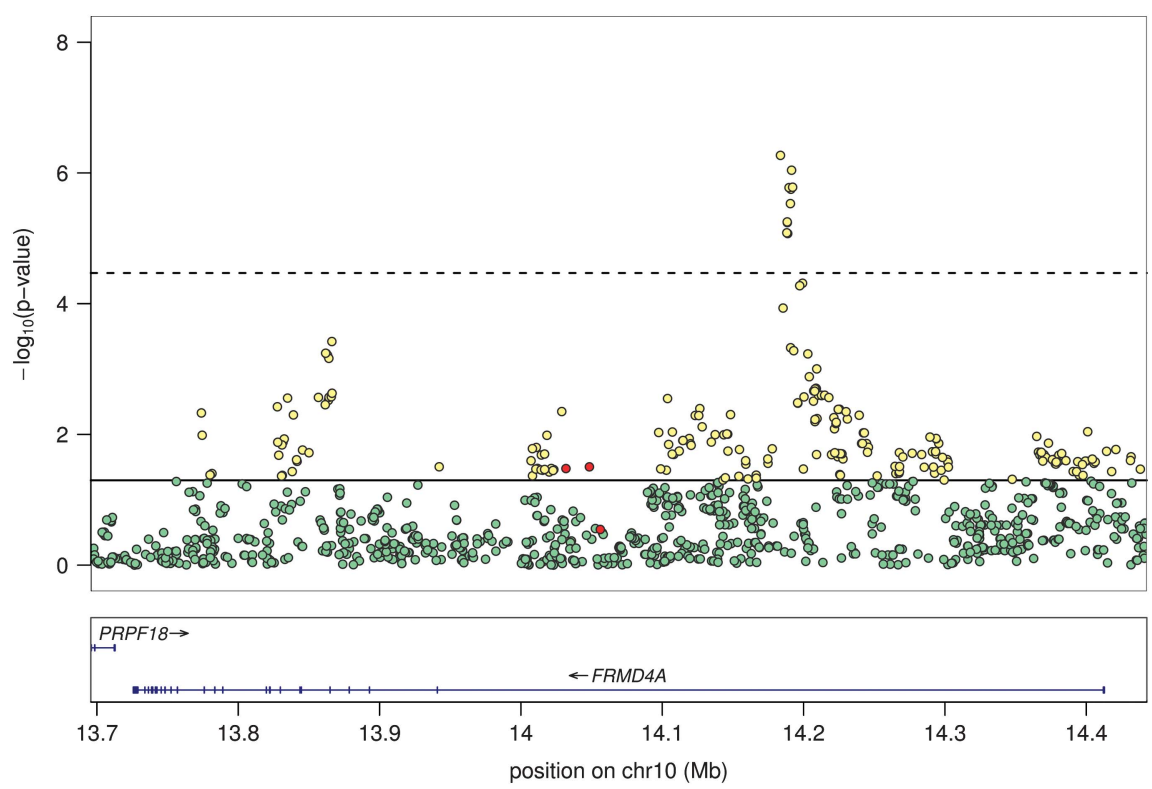

Figure 3 Association of single-nucleotide polymorphism (SNP) in the FRMD4A locus with plasma $A \beta_{1-42} / A \beta_{1-40}$ level following meta-analyses of $z$-score $\beta$ coefficients under an additive model adjusted for age and gender using three independent healthy populations. SNPs in red are nominally associated with A $\beta$ peptide levels. SNPs in are the three markers defining the AAC haplotype assocated with Alzheimer's disease (AD) risk.

variations-neither of which is captured by imputation. Interestingly, several copy-number variations within the FRMD4A locus have been described (http://projects.tcag.ca/variation/).

Little is known about the function of the protein encoded by FRMD4A in animal cells. It belongs to the FERM super family, which includes ubiquitous components of the cytocortex involved in cell structure, transport and signalling functions. ${ }^{27}$ According to a recent report, the FRMD $4 A$ gene product may regulate epithelial polarity by interacting with Arf6 and the PAR complex. ${ }^{24}$ Interestingly, several other lines of evidence suggest that Arf6 modulates cell polarity in various systems-including neurons. Arf6 reportedly regulates dendritic branching in hippocampal neurons and neurite outgrowth in PC12 cells. ${ }^{28,29}$ Finally, Arf6 was recently reported to control APP processing, ${ }^{27}$ suggesting that FRMD4A could also be implied in this metabolism. This hypothesis is sustained by the observation of an association of the FRMD $4 A$ locus with plasma $A \beta_{1-42} / A \beta_{1-40}$, at once reinforcing the plausibility of the association of this gene with AD risk and its potential implication in a subtle control of the APP metabolism. Unfortunately, as the SNPs defining the AAC haplotype were only available by imputation in the Rotterdam and CHS study, search for association of the AAC haplotype with plasma $A \beta_{1-42} / A \beta_{1-40}$ was not possible. Furthermore, it is important to keep in mind that this result is difficult to interpret in terms of AD pathophysiological process. First, it is not known whether plasma $A \beta$ peptides reflect a dynamic equilibrium between the brain, CSF and plasma compartments. ${ }^{30-34}$ Second, the source of plasma $A \beta$ species is not known and the A $\beta$ peptides' physiological functions are still not fully understood..$^{35,36}$

However, taken as a whole, these data suggest that FRMD4A could be a relevant candidate gene for $\mathrm{AD}$ risk and the basis for another possible pathophysiological pathway for AD.

\section{Conflict of interest}

The authors declare no conflict of interest.

\section{Acknowledgments}

The work was made possible by the generous participation of the control subjects, the patients and their families. This work was supported by the National Foundation for Alzheimer's disease and related disorders, the Institut Pasteur de Lille, the Centre National de Génotypage, Inserm, FRC (fondation sur la recherche sur le cerveau) and Rotary. The Three-City Study was performed as part of a collaboration between the Institut National de la Santé et de la Recherche Médicale (Inserm), the Victor Segalen Bordeaux II University and Sanofi-Synthélabo. The Fondation pour la Recherche Médicale funded the preparation and initiation of the study. The 3C Study was also funded by the Caisse Nationale Maladie des Travailleurs Salariés, Direction Générale de la Santé, MGEN, Institut de la Longévité, Agence Française de Sécurité Sanitaire des Produits de Santé, the Aquitaine and Bourgogne Regional Councils, Fondation de France and the joint French Ministry of Research/INSERM 'Cohortes et collections 
de données biologiques’ programme. Lille Génopôle received an unconditional grant from Eisai. Experiments presented in this paper were carried out using the EGI European grid infrastructure, supported by EGI, NGI France and Université Lille 1. We highly appreciate and thank the technical staff of the CRILille 1 center for their strong and helpful support. Belgium sample collection: the research at the Antwerp site was in part supported by the Interuniversity Attraction Poles program P6/43 of the Belgian Science Policy Office, the Foundation for Alzheimer Research (SAO-FRMA), a Methusalem Excellence Grant of the Flemish Government, the Research Foundation Flanders (FWO), the University of Antwerp and the Special Research Fund of the University of Antwerp, the Antwerp Medical Research Foundation and Neurosearch, Belgium. KS and KB are postdoctoral fellows of the FWO. The Antwerp site authors thank the personnel of the VIB Genetic Service Facility, the Biobank of the Institute Born-Bunge and the Departments of Neurology and Memory Clinics at the ZNA Middelheim, ZNA Hoge Beuken and the University Hospitals Leuven. Italian sample collections: the Florence site was supported by a grant from the Italian Ministry of Health: Progetto Strategico 2007- convenzione 39 (sottoprogetto 2). The Milan site was supported by grants from Italian Ministry of Health (PS39) and 'Fondazione Monzino'. We thank the expert contribution of Mr. Carmelo Romano. Spanish sample collection: the Madrid site (MB) was supported by grants of the Ministerio de Educación y Ciencia and the Ministerio de Sanidad y Consumo (Instituto de Salud Carlos III), and an institutional grant of the Fundación Ramón Areces to the CBMSO. We thank I Sastre and Dr A MartínezGarcía for the preparation and control of the DNA collection, and Drs P Gil and P Coria for their cooperation in the cases/controls recruitment. We are grateful to the Asociación de Familiares de Alzheimer de Madrid (AFAL) for continuous encouragement and help. The Gran Canaria site was supported by grants of the Ministerio de Sanidad y Consumo (Instituto de Salud Carlos III), and the Fundacion Canaria de Investigacion en Salud (FUNCIS). German sample collection: the Saarland site at the UdS was supported by the German National Genome Research Network (NGFN), grant 01GS08125 to MR, the Helmholtz Alliance for Mental Health in Ageing Societies (HelMA), and the Deutsche Forschungsgemeinschaft (DFG) grant INST 256/317-1 FUGG. Swedish sample collection: the department of medical Epidemiology and Biostatistics at the Karolinska Institute is funded by the US National Institutes of Health (AG028555, AG08724, AG 04563, AG10175, AG08861. The sample collection contributed by LF and CG were financially supported in part by the Swedish Brain Power network, the Marianne and Marcus Wallenberg Foundation, the Swedish Research Council (5212010-3134), the King Gustaf V and Queen Victoria's Foundation of Freemasons, the Regional Agreement on Medical Training and Clinical Research
(ALF) between Stockholm County Council and the Karolinska Institutet. We also wish to acknowledge Anne Kinhult-Ståhlbom, Charlotte Forsell, Håkan Thonberg and Lena Lilius for preparation of the DNA and samples.

\section{References}

1 Hardy J, Selkoe D. The amyloid hypothesis of Alzheimer's disease: progress and problems on the road to therapeutics. Science 2002 297: 353-356.

2 Small SA, Duff K. Linking Abeta and tau in late-onset Alzheimer's disease: a dual pathway hypothesis. Neuron 2008; 60: $534-542$.

3 Lambert JC, Amouyel P. Genetic heterogeneity of Alzheimer's disease: complexity and advances. Psychoneuroendocrinology 2007; 32: S62-S70.

4 Genin E, Hannequin D, Wallon D, Sleegers K, Hiltunen M, Combarros $\mathrm{O}$ et al. APOE and Alzheimer disease: a major gene with semi-dominant inheritance. Mol Psychiatry 2011; 16: 903-907.

5 Lambert JC, Heath S, Even G, Campion D, Sleegers K, Hiltunen M et al. Genome-wide association study identifies variants at CLU and CR1 associated with Alzheimer's disease. Nat Genet 2009; 41: 1094-1099.

6 Harold D, Abraham R, Hollingworth P, Sims R, Gerrish A, Hamshere ML et al. Genome-wide association study identifies variants at CLU and PICALM associated with Alzheimer's disease. Nat Genet 2009; 41: 1088-1093.

7 Seshadri S, Fitzpatrick AL, Ikram MA, DeStefano AL, Gudnason V, Boada $\mathrm{M}$ et al. Genome-wide analysis of genetic loci associated with Alzheimer disease. JAMA 2010; 303: 1832-1840.

8 Hollingworth P, Harold D, Sims R, Gerrish A, Lambert JC, Carrasquillo MM et al. Common variants at ABCA7, MS4A6A/ MS4A4E, EPHA1, CD33 and CD2AP are associated with Alzheimer's disease. Nat Genet 2011; 43: 429-435.

9 Naj AC, Jun G, Beecham GW, Wang LS, Vardarajan BN, Buros J et al. Common variants at MS4A4/MS4A6E, CD2AP, CD33 and EPHA1 are associated with late-onset Alzheimer's disease. Nat Genet 2011; 43: 436-441.

10 Lambert JC, Amouyel P. Genetics of Alzheimer's disease: new evidences for an old hypothesis? Curr Opin Genet Dev 2011; 21: 295-301.

11 Trégouët DA, König IR, Erdmann J, Munteanu A, Braund PS, Hall AS et al. Genome-wide haplotype association study identifies the SLC22A3-LPAL2-LPA gene cluster as a risk locus for coronary artery disease. Nat Genet 2009; 41: 283-285.

12 McKhann G, Drachman D, Folstein M, Katzman R, Price D, Stadlan EM. Clinical diagnosis of Alzheimer's disease: report of the NINCDS-ADRDA work group under the auspices of department of Health and Human services task force on Alzheimer's disease. Neurology 1984; 34: 939-944.

13 Mirra SS, Heyman A, McKeel D, Sumi SM, Crain BJ, Brownlee LM et al. The consortium to establish a registry for Alzheiemr's disease (CERAD). Part II. Standardization of the neuropathologic assessment of Alzheimer's disease. Neurology 1991; 41: 479-486.

14 Van Oijen M, Hofman A, Soares HD, Koudstaal PJ, Breteler MM. Plasma Abeta(1-40) and Abeta(1-42) and the risk of dementia: a prospective case-cohort study. Lancet Neurol 2006; 5: 655-660.

15 Lambert JC, Schraen-Maschke S, Richard F, Fievet N, Rouaud O, Berr C et al. Association of plasma amyloid beta with risk of dementia: the prospective three-city study. Neurology 2009; 73: 847-853.

16 Lopez OL, Kuller LH, Mehta PD, Becker JT, Gach HM, Sweet RA et al. Plasma amyloid levels and the risk of $\mathrm{AD}$ in normal subjects in the cardiovascular health study. Neurology 2008; 70: 1664-1671.

17 Mathias RA, Gao P, Goldstein JL, Wilson AF. A graphical assessment of $P$-values from sliding window haplotype tests of association to identify asthma susceptibility loci on chromosome 11q. BMC Genet 2006; 7: 38. 
$18 \mathrm{Yu} \mathrm{Z,} \mathrm{Schaid} \mathrm{DJ.} \mathrm{Sequential} \mathrm{haplotype} \mathrm{scan} \mathrm{methods} \mathrm{for} \mathrm{associa-}$ tion analysis. Genet Epidemiol 2007; 31: 553-564.

19 Huang BE, Amos CI, Lin DY. Detecting haplotype effects in genome wide association studies. Genet Epidemiol 2007; 31: 803-812.

20 Tregouet DA, Ricard S, Nicaud V, Arnould I, Soubigou S, Rosier M et al. In-depth haplotype analysis of ABCA1 gene polymorphisms in relation to plasma ApoA1 levels and myocardial infarction. Arterioscler Thromb Vasc Biol 2004; 24: 775-781.

21 Tregouet DA, Escolano S, Tiret L, Mallet A, Golmard JL. A new algorithm for haplotype-based association analysis: the stochasticEM algorithm. Ann Hum Genet 2004; 68: 165-177.

22 Tregouet DA, Garelle V. A new JAVA interface implementation of THESIAS: testing haplotype effects in association studies. Bioinformatics 2007; 23: 1038-1039.

23 Gagliardi F, Jones B, Grey F, Bégin ME, Heikkurinen M. Building an infrastructure for scientific grid computing: status and goals of the EGEE project. Philos Transact A Math Phys Eng Sci 2005; 363 . 1729-1742.

24 Ikenouchi J, Umeda M. FRMD4A regulates epithelial polarity by connecting Arf6 activation with the PAR complex. Proc Natl Acad Sci USA 2010; 107: 748-753.

25 Sannerud R, Declerck I, Peric A, Raemaekers T, Menendez G, Zhou $\mathrm{L}$ et al. ADP ribosylation factor 6 (ARF6) controls amyloid precursor protein (APP) processing by mediating the endosomal sorting of BACE1. Proc Natl Acad Sci USA 2011; 108: E559-E568.

26 Hamshere ML, Holmans PA, Avramopoulos D, Bassett SS, Blacker $\mathrm{D}$, Bertram L et al. Genome-wide linkage analysis of 723 affected relative pairs with late-onset Alzheimer's disease. Hum Mol Genet 2007; 16: 2703-2712.

27 Tepass U. FERM proteins in animal morphogenesis. Curr Opin Genet Dev 2009; 19: 357-367.

28 Hernández-Deviez DJ, Casanova JE, Wilson JM. Regulation of dendritic development by the ARF exchange factor ARNO. Nat Neurosci 2002; 5: 623-624.

29 Albertinazzi C, Za L, Paris S, de Curtis I. ADP-ribosylation factor 6 and a functional PIX/p95-APP1 complex are required for Rac1B-mediated neurite outgrowth. Mol Biol Cell 2003; 14: 1295-1307.

30 Mehta PD, Pirttila T, Patrick BA, Barshatzky M, Mehta SP. Amyloid beta protein 1-40 and 1-42 levels in matched cerebrospinal fluid and plasma from patients with Alzheimer disease. Neurosci Lett 2001; 304: 102-106.

31 Vanderstichele H, Van Kerschaver E, Hesse C, Davidsson P, Buyse MA, Andreasen $\mathrm{N}$ et al. Standardization of measurement of beta-amyloid(1-42) in cerebrospinal fluid and plasma. Amyloid 2000; 7: 245-258.

32 Kawarabayashi T, Younkin LH, Saido TC, Shoji M, Ashe KH, Younkin SG. Age-dependent changes in brain, CSF, and plasma amyloid (beta) protein in the Tg2576 transgenic mouse model of Alzheimer's disease. J Neurosci 2001; 21: 372-381.

33 Ghersi-Egea JF, Gorevic PD, Ghiso J, Frangione B, Patlak CS, Fenstermacher JD. Fate of cerebrospinal fluid-borne amyloid betapeptide: rapid clearance into blood and appreciable accumulation by cerebral arteries. J Neurochem 1996; 67: 880-883.

34 Giedraitis V, Sundelöf J, Irizarry MC, Gårevik N, Hyman BT, Wahlund LO et al. The normal equilibrium between CSF and plasma amyloid beta levels is disrupted in Alzheimer's disease. Neurosci Lett 2007; 427: 127-131.

35 Soscia SJ, Kirby JE, Washicosky KJ, Tucker SM, Ingelsson M, Hyman B et al. The Alzheimer's disease-associated amyloid betaprotein is an antimicrobial peptide. PloS One 2010; 5: e9505.

36 Lambert JC, Dallongeville J, Ellis KA, Schraen-Maschke S, Lui J, Laws $S$ et al. Association of plasma $A \beta$ peptides with blood pressure in the elderly. PloS One 2011; 6: e18536.

(c) This work is licensed under the Creative

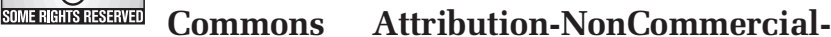
Share Alike 3.0 Unported License. To view a copy of this license, visit http://creativecommons.org/ licenses/by-nc-sa/3.0/

Supplementary Information accompanies the paper on the Molecular Psychiatry website (http://www.nature.com/mp) 